\title{
Formación a líderes acompañantes
}

\author{
Leydy Aleen Erazo y Óscar Zamora Arévalo
}

\section{Resumen}

El video resume la experiencia de una capacitación virtual sobre la permanencia y el rezago escolar, dirigida a estudiantes de semestres superiores de la de Licenciatura de la Facultad de Psicología, unAm. Los participantes hablan sobre las actividades, experiencias y testimonios, así como de su satisfacción con los contenidos abordados durante las tres semanas de entrenamiento.

La literatura especializada en el tema muestra que los estudiantes pares son una red de apoyo efectivo cuando reciben capacitación y estructura institucional. Este grupo de personas hace parte de las estrategias institucionales para mitigar el rezago escolar, en el contexto del covid-19.

Esta actividad fue financiada por un proyecto PAPIME-UNAM autorizado en 2017 que finaliza en 2021.

Palabras clave: enseñanza, permanencia, rezago educativo, psicología.

\section{TRAINING FOR ACCOMPANYING LEADERS}

\begin{abstract}
The video summarizes the experience of a virtual training on school permanence and student attrition, aimed at students in higher semesters of the Bachelor's Degree in the Faculty of Psychology, unAm. The participants talk about the activities, experiences and testimonies, as well as their satisfaction with the contents addressed throughout three weeks of training.

The specialized literature on this subject shows that peer students serve as an effective support network when they receive training and institutional structure. This group of people is part of the institutional strategies purported to mitigate withdrawal, in the context of Covid-19.
\end{abstract}

This activity was financed by a PAPIME-UNAM project authorized in 2017 that ends in 2021.

Keywords: teaching, permanence, educational lag, psychology.

Recepción: 30/10/2020. Aprobación: 1/12/2020.

Dol: http://doi.org/10.22201/cuaieed.16076079e.2021.22.1.20

Universidad Nacional Autónoma de México, Coordinación de Universidad Abierta, Innovación Educativa y Educación a Distancia (CUAIEED) Este es un artículo de acceso abierto bajo la licencia de Creative Commons 4.0 (c) (i) (2) 
"Formación a líderes acompañantes" Leydy Aleen Erazo y Óscar Zamora Arévalo

Linkedin: Leydy Aleen Erazo

Twitter: @aleenerazo

Docente y Secretaria Académica para la Licenciatura en de la Facultad de Psicología de la Universidad Nacional Autónoma de México (UNAM).

Sus campos de interés están enfocados en la educación superior, por un lado, en la evaluación educativa como medio de transformación, y por otro, en la mejora de la permanencia y la disminución del abandono escolar. Estudió Psicología en la Universidad Konrad Lorenz en Bogotá-Colombia y la maestría en evaluación educativa en la unam.

\section{Óscar Zamora Arévalo}

arevalo@unam.mx

Profesor Titular "B" de Tiempo Completo Definitivo y jefe de la División de Estudios Profesionales de la Licenciatura en la Facultad de Psicología de la Universidad Nacional Autónoma de México (UNAM).

Sus campos de interés están enfocados en la cognición comparada y evaluación educativa, por un lado, en la cognición comparada tanto con organismos humanos y su desarrollo cognitivo y con organismos no humanos su interés ha sido en proponer modelos de aprendizaje y procesos cognitivos básicos. En la evaluación educativa su interés se ha enfocado en analizar la validez de constructo, la confiabilidad y la calidad técnica en general de los instrumentos de valoración para los procesos de selección en educación básica y media superior, así como en la mejora de la permanencia y la disminución del abandono escolar. Estudió la Licenciatura en Psicología, la Maestría en Análisis Experimental de la Conducta y el Doctorado en la Facultad de Psicología en la UNAM. 


\section{¿Qué racei os en la fcrr. adc ón?}

\section{Sitios de interés}

- Tercera Conferencia Latinoamaericana sobre el Abandono en la Educación Superior. (s. f.). CLABES. http://clabes-alfaguia.org/ clabes-2013/comunicaciones_tema1.php

- Murillo, D. (2019, 12 febrero). Memoria Sexta Conferencia Latinoamericana sobre el abandono en la Educación Superior, VI CLABES. UTP-Ridda. https://ridda2.utp.ac.pa/ handle/123456789/6121?show=full

- Universidad Tecnológica de Panamá. (2018). Memoria Octava Conferencia Latinoamericana sobre el abandono en la Educación Superior. Editorial Universidad de Santiago de Chile. https://www. mentoriaiberoamerica.org/wp-content/uploads/2020/04/CLABES_ VIII.pdf

\section{Cómo CITAR ESTE ARTículo}

* Erazo, Leydy Aleen y Zamora Arévalo, Óscar. (2021, enero-febrero). Formación a líderes acompañantes. Revista Digital Universitaria (RDU), 22(1). Dol: http://doi. org/10.22201/cuaieed.16076079e.2021.22.1.20 